\title{
Investigation on Thermal Aging of HVDC XLPE
}

\author{
Weiwei $\mathrm{LI}^{1, \mathrm{a}^{*}}$, Qiang SH${ }^{2, \mathrm{~b}}$, and Wei Xiao ${ }^{1}$ \\ ${ }^{1}$ State Grid Sichuan Electric Power Research Institute, Chengdu, 610000, China \\ ${ }^{2}$ Sichuan Electric Power Corporation Metering Center, Chengdu, 610000, China \\ aemail: liweiwei0830@163.com, bemail:awaityou1986@gmail.com
}

\begin{abstract}
Keywords: Cross-linked polyethylene, thermal aging, assessment techniques, activation energy. Abstract. HVDC XLPE cables were widely used in the transmission line for the excellent characteristics. The thermal aging properties of HVDC XLPE insulation were investigated in this work, based on different assessment techniques, such as IR Prestige-21 Infrared Spectrometer, Thermo-Gravimetric Analyzer, and X-Ray Diffractometer. It was found that, the molecular structure of XLPE cable insulation changed during the thermal aging process, carbonyl deposed as the increase of aging time and the increase of aging temperature. There was one fastest decomposition temperature for un-aged specimen and specimen aged at low temperature, while there were multiple decomposition temperatures for specimens aged at high temperature. Numerous new chemical bonds, whose activation energies differ from that of XLPE, arise during the high temperature aging. The XLPE crystallinity increased during low-temperature aging while decreased during high-temperature aging. Low-temperature is conducive to the transition of amorphous region into crystallization region, while high-temperature has damaging effect on the crystallization region.
\end{abstract}

\section{Introduction}

HVDC XLPE cables were widely used in the transmission line for the excellent properties. The XLPE cables were used for more than 20 years in quiet a lot cities. It is of great importance to assessment the state of these cables in operation for a long time.

Aging which is difficult to be directly observed, needs to use all kinds of assessment techniques to research [1-5]. ARTEMIS team found that the total porosity decreased slightly, while the total pore surface area increased, considered that the pores grew and were connected to each other after aging [1]. Mazzanti G [2] considered that the degradation of cable insulation starting from the micro pores in the materials. Laurent $C[3,4]$ used EL technology to characterize the energy released in the aging process, so as to determine the starting and the development speed of the aging process. Zhang GJ [6] found that the EL starting voltage and EL density of aged LDPE specimens were lower than that of un-aged specimen.

At home and abroad, the study on the aging characteristics of XLPE cable insulation has been carried out for a long time, but there are still many problems in the current theories [7, 8]. Polymer during aging process is in a non equilibrium state. Changes occur in the aging process, which can affect the physical chemical properties, electrical properties, thermal properties of polymers. This work focused on the evolution law of aggregate state structure and trap parameters of thermal aged cable insulation.

\section{Experiment Methods and Devices}

\section{Sample Preparation}

Current in inner conductor will produce large amount of heat in cables in service, this heat will diffuse outward along the radial direction of cable slowly. Therefor, heat is one of the important aging factors of cable in long-term operation. In this work, 220kV XLPE cable was selected to be peeled into $500 \mu \mathrm{m}$ sheets for thermal aging. According to IEC-60811-1-2(2000-07), cable sheets were aged at $100^{\circ} \mathrm{C}, 120^{\circ} \mathrm{C}, 140^{\circ} \mathrm{C}$ [9]. 


\section{Test Systems}

IR Prestige-21 Infrared Spectrometer was used for the FTIR test of specimens. There is a corresponding relationship between molecular groups and infrared absorption bands. Infrared Spectrometer records the relationship between the infrared transmittance and the wave number, and the FTIR curve can provide the molecular vibrational spectra to explore the molecular structure.

The DX-1000 X-Ray Diffractometer was used for the XRD test of specimens, the scan rate was $0.3 \% \mathrm{~min}$, and scanning angle were $15^{\circ} \sim 30^{\circ}$.

The TGA/SDTA $851^{\mathrm{e}}$ Thermo-Gravimetric Analyzer was used to conducts thermo-gravimetric analysis on the specimens in $\mathrm{N}_{2}$ atmosphere. The test temperature range was from $50^{\circ} \mathrm{C}$ to $600^{\circ} \mathrm{C}$, the heating rate was $10^{\circ} \mathrm{C} / \mathrm{min}$, and the test data was recorded automatically by a computer.

\section{Experimental Results and Discussion}

\section{FTIR}

Aging can cause chemical changes in the cable insulation, Fourier transform infrared spectroscopy can be used to study changes in the molecular chain structure of the material. In order to reveal the chemical defects trends with aging, the molecular structure of aged XLPE cable insulation was investigated by FTIR in this work. Fig1 shows the FTIR of thermal aged XLPE cable insulation. Clearly, carbonyl characteristic absorption peaks at $1700 \sim 1750 \mathrm{~cm}^{-1}$ changed in the thermal aged XLPE cable insulation compared with the un-aged one. This wave bond includes PE oxidation degradation products,such as, aldehyde $\left(-\mathrm{CHO}\right.$ at $\left.1708 \mathrm{~cm}^{-1}\right)$, ketone $\left(-\mathrm{CH}_{2}-\mathrm{CO}-\mathrm{CH}_{2}-\right.$ at $\left.1715 \mathrm{~cm}^{-1}\right)$ and ester $\left(-\mathrm{CH}_{2}-\mathrm{CO}-\mathrm{O}-\mathrm{CH}_{2}-\right.$ at $\left.1735 \mathrm{~cm}^{-1}\right)$.
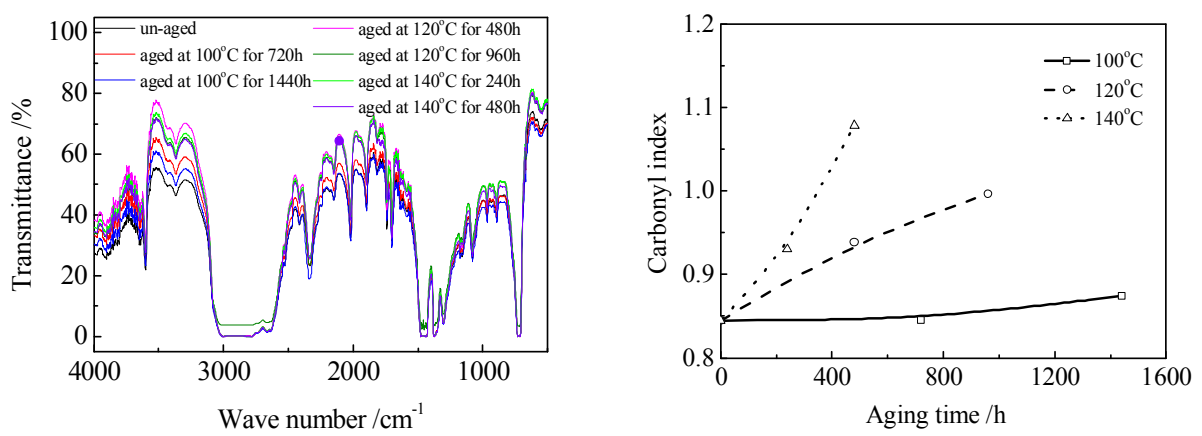

(a) FTIR curve

(b) Carbonyl index as a function of aging time

Fig.1. FTIR of thermal aged XLPE cable insulation

In order to quantitatively analysis the change of molecular structure after aging, internal standard method was introduced in this work to exclude the impact of specimen thickness on test results. The internal standard peak at $2010 \mathrm{~cm}^{-1}$ is co-frequency vibration peak of peaks at $1303 \mathrm{~cm}^{-1}$ and $719 \mathrm{~cm}^{-1}$. This peak area has nothing to do with specimen crystallinity and content of thermal aging product. Carbonyl index is defined as follows,

Carbonyl index $=A_{\text {Carbonyl peak }} / A_{\text {Standard peak }}$

Where, $A_{\text {Standard peak }}$ refers to the area of standard peak at $2010 \mathrm{~cm}^{-1}, A_{\text {Carbonyl peak }}$ refers to area of carbonyl absorption peak at $1700 \mathrm{~cm}^{-1} \sim 1750 \mathrm{~cm}^{-1}$.

According to the equation, the carbonyl index as a function of aging time is shown in Fig.1(b). Thermo-oxidative aging occurred at three temperatures, the carbonyl index increased with the aging time during thermal aging process. The carbonyl index increased slowly after 1440 hours thermal aging at $100^{\circ} \mathrm{C}$. As the aged temperature increased to $120^{\circ} \mathrm{C}$, the growth rate of carbonyl index increased. As for $140^{\circ} \mathrm{C}$ thermal aging, the carbonyl index increased fastest. FTIR results show that, XLPE cable insulation aging is co-effect of aging time and aging factors. The molecular structure of XLPE cable insulation changed during the thermal aging process. The difference between the high temperature thermal aging and low temperature thermal aging is the growth rate of carbonyl, the increase of aging temperature will significantly accelerate the XLPE thermal oxygen aging rate. 
The cable generates heat during operation, and the conduction of heat in cable is slow, leading to the temperature rise in XLPE insulation. The XLPE macr-molecular chains will be oxidized into hydrogen peroxides on the effects of long-term heat under aerobic environment. Hydrogen peroxide was easily to decompose into free radicals. The free radical will be oxidized into peroxide radicals in the case of oxygen, and it also can generate hydrogen peroxide and a new free radical through extracting hydrogen atom from other polymers. The above reactions can cycle, producing a large number of carbonyl chemical trap, expressed as follows:

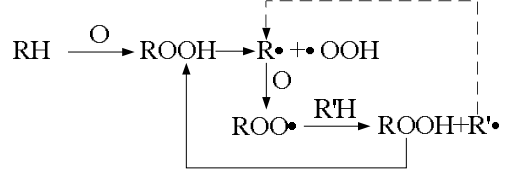

The rise of temperature will make it easier for the above reaction process, and will promote the oxygen into the space between the molecular chains, leading to oxygen uptake of chains increasing greatly. And therefore carbonyl index at high-temperature thermal aging is significantly greater than that at low-temperature thermal aging process.

\section{TG}

The TG curves of thermal aged specimens are shown in Fig.2, and the DTG curves, that are first derivative of TG curves, are shown in the illustration of Fig.2. It can be seen that, the decomposition temperature of XLPE cable insulation decreased after aging, and thermal stability of aged specimen decreased. Meanwhile, there was one peak in the DTG curves of un-aged specimen and aged at low temperature, that was, there was one the fastest decomposition temperature. There are multiple peaks in the DTG curves for specimens aged at high temperatures. According to the FTIR, numerous new chemical bonds occurred during high temperature aging process. The activation energies of these chemical bonds differ from each other, that is why specimens aged at high temperature present multiple decomposition temperatures. The activation energy of chemical bonds $\mathrm{C}-\mathrm{C}, \mathrm{C}-\mathrm{H}$ and $\mathrm{C}$ $-\mathrm{O}$ are $3.50 \mathrm{eV}, 3.55 \mathrm{eV}$ and $3.28 \mathrm{eV}$ respectively. The higher the thermal decomposition temperature and activation energy are, the better the thermal stability.

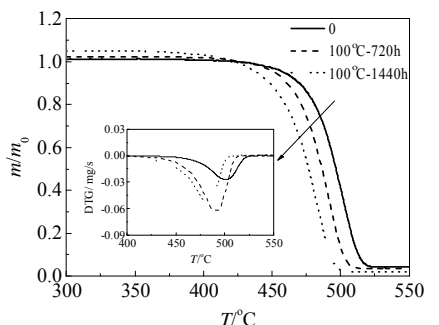

(a) $100^{\circ} \mathrm{C}$ thermal aging

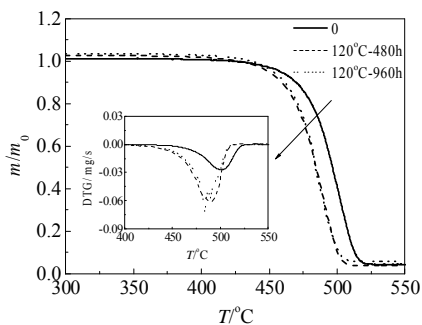

(b) $120^{\circ} \mathrm{C}$ thermal aging

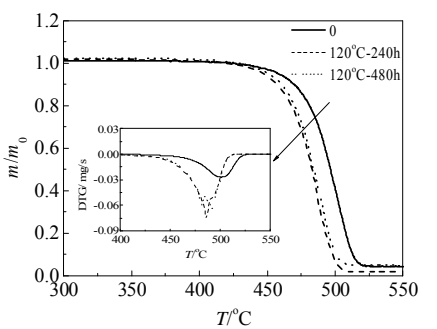

(c) $140^{\circ} \mathrm{C}$ thermal aging

Fig.2. TG curve of thermal aged XLPE cable insulation

\section{XRD}

X-ray diffraction was used to analyze the crystalline morphology of XLPE cable insulation, and to calculate the degree of crystallization. Fig. 3 shows the XRD curve of thermal aged XLPE cable insulation, the abscissa indicates the scan angle, the longitudinal coordinates refers to the diffraction intensity. The actual measuring XRD curve were separated by Gaussion method, solid line in the Fig. 3 refers to the test curve, and the dotted line is the fitted curve by Gaussion method.

There are two diffraction peaks in the XRD curve of XLPE cable insulation which located in regions $21.4^{\circ} \sim 21.6^{\circ}$ and $23.7^{\circ} \sim 24^{\circ}$ corresponding to XLPE orthogonal crystal lattice plane (110), (200), respectively. The dispersing diffraction peaks crossover $15^{\circ} \sim 26^{\circ}$ is corresponded to the crystalless state of aggregation. It was also found that, the diffraction peak corresponding to orthogonal crystal lattice plane (200) is sensitive to thermal aging temperature. The diffraction peaks around $24^{\circ}$ increase obviously as the increase of aging time at $100^{\circ} \mathrm{C}$. The area of peak around $24^{\circ}$ 
after aging 60 days even is greater than that of peak around $21.5^{\circ}$. This indicated that, the macro-molecular along the chain direction is arranged more neatly during the process of low temperature heat aging. The diffraction peaks around $24^{\circ}$ increase obviously as the decrease of aging time at $120^{\circ} \mathrm{C}$ and $140^{\circ} \mathrm{C}$.

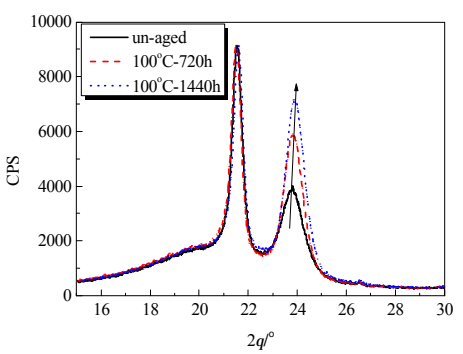

(a) $100^{\circ} \mathrm{C}$ thermal aging

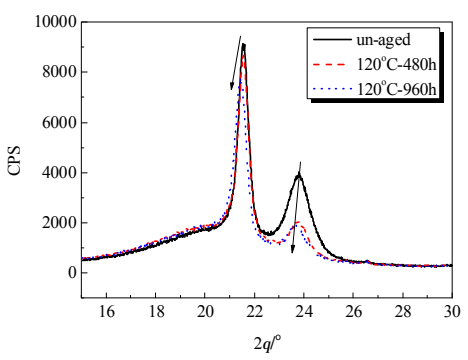

(b) $120^{\circ} \mathrm{C}$ thermal aging

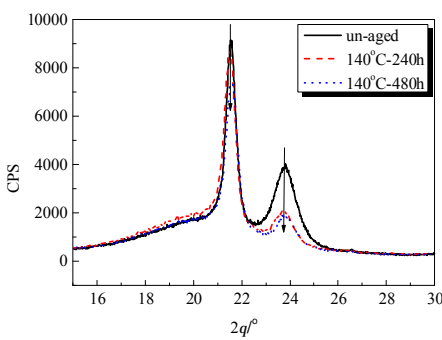

(c) $140^{\circ} \mathrm{C}$ thermal aging

Fig.3. Gaussian peak fitting for XRD curve of thermal aged XLPE cable insulation

Diffraction phenomenon will occur when the X-ray inject into the crystal. XLPE is semi-crystalline polymer, whose crystalline phase can produce X-ray diffraction phenomenon. X-ray diffraction is effective method to investigate the crystal structure. The crystallinity can be calculated through the XRD curve. This crystallinity calculated method considers the diffraction phenomenon of crystallinity and dispersing diffraction phenomenon of amorphous regions. The degree of crystallinity calculated based on the area of each peak. The XRD curve can be fitted by Gaussion peak separation, as shown in the inset of Fig.4. The XRD curve of XLPE can be can be regarded as a superposition of three peaks, peak 1 corresponds to amorphous peak, peak 2 and peak 3 correspond to the crystal peaks. Therefor the XLPE crystallinity can be expressed as:

$C=\left(S_{2}+S_{3}\right) /\left(S_{1}+S_{2}+S_{3}\right)$

Where, $S_{1}, S_{2}, S_{3}$ refer to the areas of peak 1, 2, 3, respectively.

According to the above equation, Fig. 4 shows the crystallinity of XLPE as functions of aging time at different aging temperatures. The XLPE crystallinity increases during low-temperature aging while decreased during high-temperature aging. The aging temperature affected the XLPE aggregation structure significantly. Low-temperature is conducive to the transition of amorphous region into crystallization region, while high-temperature has damaging effect on the crystallization region.

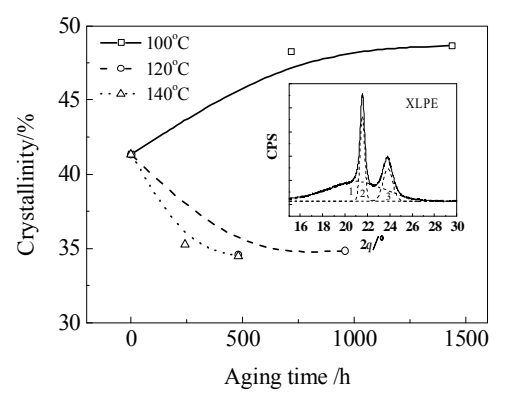

Fig.4. Crystallinity of thermal aged specimen as function of aging time

Degradation reactions as well as crosslinking reactions occur in XLPE during aging process. Free radicals crosslinking react with each other, the crosslinking process can be expressed as follows:

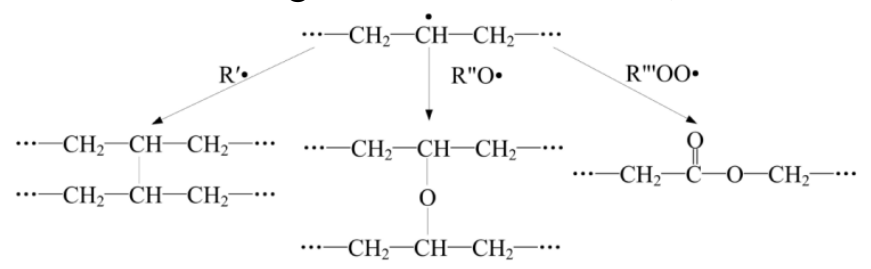

Crosslinking reactions are dominant during low temperature thermal aging process, and the degree of crystallinity increases. As for high temperature thermal aging process, the rupture of XLPE 
polymer main chain speed up, more and more chain scission and oxidation products are produced, leading to the transition of crystalline to amorphous and the decrease of crystallization degree.

\section{Conclusion}

This wok focus on the assessment of thermal aged cable insulation.

(1) The molecular structure of XLPE cable insulation changed during the thermal aging process. Carbonyl produced in aging process, the carbonyl index at high-temperature thermal aging is significantly greater than that at low-temperature thermal aging process.

(2) There is one the fastest decomposition temperature for un-aged specimen and aged at low temperature, while there are multiple decomposition temperatures for specimens aged at high temperature. Numerous new chemical bonds occur during the high temperature, and the activation energy to break different chemical bonds is different.

(3) The aging temperature affected the XLPE aggregation structure significantly. The XLPE crystallinity increases during low-temperature aging while decreased during high-temperature aging. Low-temperature is conducive to the transition of amorphous region into crystallization region, while high-temperature has damaging effect on the crystallization region.

\section{Acknowledgements}

This work was supported by the work Investigation on the Assessment Techniques and Device of Cable Joint and Cable Insulation.

\section{References}

[1] Fothergill JC, Montanari GC, Stevens GC, et al. Electrical, microstructural, physical and chemical characterization of HV XLPE cable peelings for an electrical aging diagnostic data base[J]. IEEE Transactions on Dielectrics and Electrical Insulation, 2003, 10(3): 514-527.

[2] Mazzanti G, Serra S, Montanari GC. Theory of inception mechanism and growth of defect-induced damage in polyethylene cable insulation[J]. Journal of Applied Physics, 2005, 98(3): 034102.

[3] Li JY, Zhao XT, Yin GL, et al. The effect of accelerated water tree ageing on the properties of XLPE cable insulation[J]. IEEE Transactions on Dielectrics and Electrical Insulation, 2011, 18(5): 1562-1569.

[4] Chen G, Fu M, Liu XZ, et al. AC aging and space-charge characteristics in low-density polyethylene polymeric insulation[J]. Journal of Applied Physics, 2005, 97(8): 083713.

[5] Densley J. Ageing mechanisms and diagnostics for power cables - An overview[J]. IEEE Electrical Insulation Magazine, 2001, 17(1): 14-22.

[6] Zhang GJ, Yang K, Tu DM, et al. Space charge and electroluminescence characteristics of thermally aged LDPE films[J]. Applied Surface Science, 2008, 255(5): 2735-2739.

[7] K. C. K. Dielectric Phenomena in Solids[M]: Elsevier Academic Press, 2004.

[8] Bamji SS, Bulinski AT, Chen Y. Thermally stimulated current technique to evaluate polymer degradation due to water treeing[J]. IEEE Transactions on Electrical Insulation, 1993, 28(2): 299-302.

[9] IEC-60811-1-2(2000-07) Common test methods for insulating and sheathing materials of electric cables and optical cables[S]. 